\title{
CAMBIOS RECIENTES EN EL SECTOR VITIVINÍCOLA: EL CASO DE LAS DENOMINACIONES DE ORIGEN ZARAGOZANAS ${ }^{1}$
}

\author{
B. Loscertales Palomar, A. I. Escalona Orcao y E. Climent López \\ Universidad de Zaragoza \\ bloscer@unizar.es; aescalon@unizar.es; ecliment@unizar.es
}

\begin{abstract}
Resumen: El sector vitivinícola ha experimentado cambios importantes en los últimos años respecto a la calidad del vino, los métodos de producción, los mercados y el tejido productivo. En este artículo se analiza la respuesta que han dado a dichos cambios las tres denominaciones de origen de la provincia de Zaragoza (Cariñena, Campo de Borja y Calatayud), que se han orientado hacia la producción de vinos de gran calidad y singularidad. Las dos primeras, apoyadas en la solidez de su tejido productivo, han evolucionado favorablemente aplicando estrategias productivas diferentes (renovación frente a tradición), mientras que la tercera, con un tejido productivo más atomizado, hace frente a un marcado proceso de ajuste.
\end{abstract}

Palabras clave: Sector vitivinícola, Denominación de Origen, Zaragoza.

\begin{abstract}
Wine industry has undergone important changes in last years concerning wine quality, production methods, markets and industrial organisation. In this article the response given by the three Protected Designations of Origin in the Zaragoza province (Cariñena, Campo de Borja and Calatayud) is analysed. They have addressed the production of great quality and singularity wines. First and second of them, with a strong productive network, have evolved positively, developing differ-
\end{abstract}

\footnotetext{
Recibido: 29-10-10. Aceptado: 3-11-11.

${ }^{1}$ El artículo presenta resultados de dos proyectos de investigación: 1) "Nuevos retos para el desarrollo territorial: los espacios del vino y del calzado en la provincia de Zaragoza", que resultó seleccionado en la I Convocatoria de los Premios de Investigación Zaragoza Provincia. Cuarto Espacio, de la Diputación Provincial de Zaragoza; 2) "Gobernanza, innovación y convenciones en las comarcas vitivinícolas de Aragón: tipología y prospectiva de Denominaciones de Origen a partir de la teoría de los mundos de producción" (CSO2008-05793-CO3-02), financiado por el Ministerio de Ciencia e Innovación.
} 
ent production strategies (renovation versus tradition), while third, with a more atomised productive network, faces a strong adjustment process.

Keywords: Wine industry, Protected Designation of Origin, Zaragoza (Spain).

\section{Introducción}

La vitivinicultura es una actividad conocida y practicada en el ámbito mediterráneo desde hace milenios. Ello explica el fuerte arraigo de la misma en el ámbito aragonés y muy especialmente en la provincia de Zaragoza, dadas sus condiciones naturales y humanas (Escalona, Loscertales y Climent, 2009). Ni el cultivo de la vid ni la elaboración del vino han cambiado, en lo esencial, desde su inicio, ya que se trata de procesos en los que la naturaleza impone sus leyes. Sin embargo, son muchos los cambios que se han venido sucediendo a lo largo de la historia y muy especialmente en las últimas décadas. Éstos responden a los retos a que se enfrentan las zonas productoras de vino y pueden ordenarse tomando como guía lo que Schumpeter denominaba "la realización de nuevas combinaciones, es decir, un nuevo artículo, un nuevo método de producción, un nuevo mercado, una nueva fuente de suministro y una nueva organización industrial" (Hall y Preston, 1990, p. 35).

Los vinos actuales no son nuevos artículos, pero ciertamente no son iguales que los que se producían con anterioridad. Recientemente, la diferenciación del producto, a partir de la incorporación en el proceso de vinificación de distintas variedades de uva y mediante diversos coupages entre las mismas, constituye un objeto de atención prioritaria; de este modo, la innovación se traduce en la producción de diferentes tipos de vinos con nuevas propiedades organolépticas y con graduaciones alcohólicas controladas. Los métodos de producción siguen basándose en un proceso natural, la fermentación del mosto de la uva, pero los procedimientos e instrumentos de control del proceso han alcanzado un elevado nivel de sofisticación tecnológica, paralelo al grado cada vez mayor de cualificación del personal técnico implicado (Albisu, 2005). Lo mismo cabe decir del cultivo de la vid, donde se desarrollan nuevas técnicas, como el cultivo en espaldera, que sustituye al tradicional en vaso, el riego con sistemas muy sofisticados o el control de la vendimia, entre otras.

Los cambios más radicales se han producido en los mercados. Si el vino antes era un producto de consumo popular, típico de la dieta mediterránea, que aportaba energía barata para el desarrollo de trabajos de fuerte desgaste físico, hoy es cada vez más un producto dirigido a un consumidor exigente que disfruta con él. Este consumidor 
conoce los vinos, los valora y demanda las nuevas propiedades ya citadas y exige además unos estándares de calidad sólo posibles con la aplicación de tecnologías avanzadas. Este cambio ha obligado a evolucionar a los productores de los países mediterráneos, como España, desde una producción tradicional de vinos baratos para el mercado popular del propio país, hacia la producción de vinos de mayor calidad orientados a satisfacer las demandas múltiples de los diferentes segmentos de los consumidores, no sólo del mercado interior, sino de cualquier otra parte del mundo.

En el caso del vino no puede pensarse en nuevas fuentes de suministro en el sentido material del término, dado que la uva es la única materia prima posible y su carácter perecedero hace inviable su traslado a largas distancias, por lo que las bodegas que elaboran el vino tienen que ubicarse próximas a los viñedos de que se abastecen. No obstante, sí puede afirmarse la existencia de las nuevas fuentes de suministro que han venido apareciendo en las últimas décadas en nuevos países productores situados en otras áreas geográficas del mundo con condiciones ambientales similares a las de la Europa mediterránea, como Sudáfrica, Estados Unidos, Chile, Argentina, Australia y Nueva Zelanda. En todos ellos el cultivo de la vid y la elaboración del vino se han desarrollado notablemente. Estos nuevos países productores están planteando una dura competencia a los países tradicionales en el mercado internacional, sobre todo alli donde se ha producido recientemente un incremento del consumo del vino. A ello cabe añadir que la superficie de viñedo desciende en los países mediterráneos europeos, lo que equivale a decir en los tradicionales productores y consumidores de vino, mientras que paralelamente se incrementa en los nuevos países productores, dándose de este modo una tendencia decreciente de la participación de aquéllos en la producción mundial de vino y creciente de éstos (Escalona, Loscertales y Climent, 2009, p. 17; Ponte, 2009, p. 238).

Los citados cambios han influido en la organización industrial del sector, cuyo tejido empresarial se presenta fuertemente diferenciado y segmentado. En ocasiones se da una clara separación entre los viticultores que producen la uva y las bodegas que elaboran el vino, pero es cada vez más frecuente que las bodegas traten de contar en su totalidad o al menos en parte con una producción agraria propia para facilitar la trazabilidad de todo el proceso productivo; ello supone en muchas ocasiones que el viñedo y la bodega estén unidos tanto en el espacio como bajo la misma dirección empresarial. Tengan o no explotación agraria propia, las empresas pueden ser pequeñas, como es el caso de las de carácter familiar o de las sociedades limitadas. Las empresas mayores están constituidas como sociedades anónimas y cooperativas; estas últimas han sido y siguen siendo muy importantes en muchos territorios vitivinícolas. Todavía pueden encontrase pequeños viticultores que elaboran su propio vino en una bodega artesanal contrastando con la gran empresa, incluso, en ocasiones, de carácter multinacional, ya que éstas están cada vez más presentes en diversas áreas del mundo (Barco, Pinillos y Navarro, 2006). 
En los países productores tradicionales, muy especialmente en el ámbito mediterráneo europeo, existe la figura de la Denominación de Origen Protegida (DOP) y otras afines, en las que se agrupan viticultores y bodegas de unas áreas geográficas determinadas. Sus consejos reguladores se encargan de garantizar que el producto con DOP utiliza sólo las variedades de uva, autóctonas o no, autorizadas para la producción del vino y acordes con las condiciones naturales y el saber hacer tradicional propio de la zona y que además cumple unos requisitos de calidad claramente establecidos (Barco, 2007). De este modo, contar con una DOP, por una parte, limita la libertad de las empresas en ella integradas, pero, por otra, les ofrece el paraguas protector $\mathrm{o}$, si se prefiere, la palanca impulsora de una imagen colectiva, aunque no siempre este paraguas es percibido favorablemente por el conjunto de las empresas incluidas en una DOP (Loscertales, 2009). En los nuevos países productores, por el contrario, no existe esta figura.

La adopción de las innovaciones que componen esta nueva combinación, descrita a grandes rasgos, constituye auténticos retos para las empresas y para las zonas productoras. Este trabajo tiene por objeto mostrar cómo se están afrontando en las tres DOP localizadas en la provincia de Zaragoza: Cariñena, Campo de Borja y Calatayud (figura 4$)^{2}$

\section{Productos y procesos: diferenciación y mejora de la calidad}

En el actual contexto internacional los productores de vino intentan conseguir productos de calidad que los diferencien de la competencia; una de las vías es el manejo adecuado de las diferentes variedades de vid. La tradicionalmente más cultivada en Aragón, donde probablemente tiene su origen, y especialmente en las tres DOP de la provincia de Zaragoza, es la Garnacha tinta; se trata de una especie que resiste bien el frío y la sequía, por lo que está perfectamente adaptada a las condiciones medioambientales de la región. Los vinos blancos siempre han tenido en esta área una producción más reducida, siendo Macabeo la uva blanca tradicionalmente utilizada; probablemente es originaria de Aragón y se conoce también con el nombre de Viura. Ambas especies están muy extendidas por el resto de España. Entre las variedades no autóctonas de uvas tintas introducidas en las últimas décadas cabe citar la Tempranillo, que se cultiva en Aragón desde hace ya algún tiempo, y otras importadas más recientemente de Francia, como Cabernet, Merlot o Syrah, si bien el origen más probable de esta última es Oriente Medio.

\footnotetext{
${ }^{2}$ Las estrategias productivas de las empresas de la DOP Calatayud han sido analizadas en un trabajo reciente en el que se comparan con las desarrolladas por empresarios de empresas de calzado (Escalona, Loscertales y Climent, 2010).
} 
Atendiendo a la proporción de viñedo que ocupan actualmente las variedades señaladas, la Garnacha sigue siendo la dominante en las tres DOP, aunque hay una diferencia significativa entre Cariñena, donde se ha reducido a una tercera parte del área vitícola, y el Campo de Borja y Calatayud, donde ocupa en torno a las dos terceras partes de la misma (figura 1); de hecho, en la DOP Cariñena las uvas tintas alóctonas ocupan actualmente el 46 por ciento de la superficie vitícola, extensión mayor que la de Garnacha. La única variedad de uva blanca bien representada en las tres DOP citadas es la Macabeo, que en valores porcentuales sobre el total de has supone el 15 por ciento del viñedo de la DOP Cariñena, el 8 de la de Calatayud y el 3 de la de Campo de Borja. El resto de la superficie, la ocupan variedades minoritarias, entre las que destaca la Mazuela, también llamada Cariñena por ser originaria del territorio que le da el nombre.

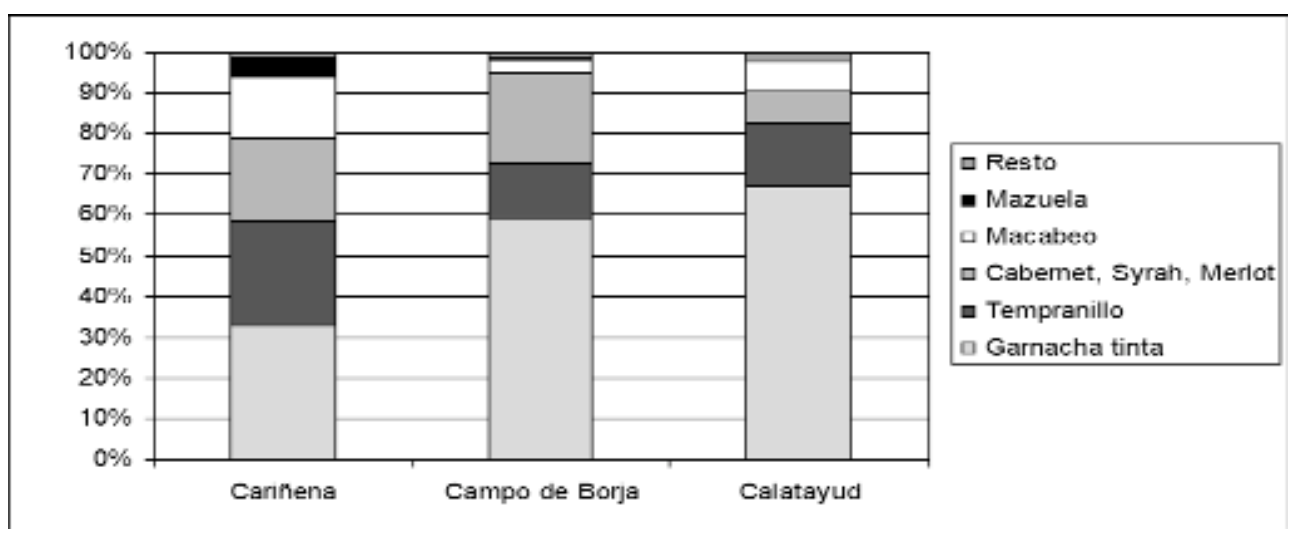

Fuente: Gobierno de Aragón (2009).

Figura 1. Distribución de la superficie de viñedo en las Denominaciones de Origen Protegidas de la provincia de Zaragoza, según las variedades de uva cultivadas.

Teniendo en cuenta la tradición común a las tres DOP, del gráfico puede deducirse la existencia de dos modelos según la diferenciación del producto; el de Cariñena, por una parte, y el de Campo de Borja y Calatayud, por otra. Los dos buscan la obtención de vino de mayor calidad, pero siguiendo procedimientos distintos: la diversificación y renovación, en el primer caso, la pervivencia en la tradición, en el segundo. El modelo de Cariñena se caracteriza por el cultivo creciente de nuevas variedades: como se ha visto, las uvas tintas autóctonas ocupan actualmente menos extensión que las alóctonas. Utilizando una serie temporal de 15 años, Loscertales (2009, p. 133) muestra que el porcentaje de Garnacha tinta en la producción total de uva se ha reducido de forma continua desde 1998. Por el contrario, el de Tempranillo 
ha ido creciendo continuamente desde 1993, así como el de las variedades de origen francés, si bien en este caso se trata de un fenómeno más reciente.

Calatayud y Campo de Borja siguen apostando por la Garnacha tradicional. Campo de Borja tiene cultivado con esta variedad casi el 60 por ciento de su viñedo y su lema es "El Imperio de la Garnacha"; además, el 40 por ciento de la superficie ocupada por esta variedad corresponde a viñas viejas, de más de 30 años de antigüedad. En Calatayud corresponde a la Garnacha casi el 70 por ciento del viñedo total, estando ocupado por viñas viejas el 58 por ciento de ese porcentaje.

Aunque las cifras son suficientemente contrastadas como para diferenciar dos modelos, la proporción de has de viñedo plantadas con variedades foráneas -un 36 por ciento en el caso del Campo de Borja y un 23 por ciento en el de Calatayudindica que en estas DOP tampoco se excluye la elaboración de vinos a partir de estas variedades importadas de otros lugares. Del mismo modo, no puede olvidarse que en Cariñena, la uva Cariñena, a la que el territorio ha dado su nombre, ocupa casi el 5 por ciento del viñedo, lo cual revela la permanencia y el interés por conservar la tradición; en esta misma línea, se puede señalar la recuperación de una variedad local de uva casi olvidada, Vidadillo o Crespiello, sobre la que investigan y ensayan enólogos, viticultores y bodegas, con el apoyo del propio Consejo Regulador, habiéndose obtenido ya algunos vinos de calidad (Loscertales, 2009, p. 126). La incorporación de nuevas variedades de uva y la selección de uvas de viñas viejas tienen por objeto conseguir nuevas propiedades organolépticas para que los vinos sorprendan y agraden a paladares exigentes, tanto si se elaboran a partir de una sola variedad, como si se hace combinando dos o más.

Las nuevas técnicas utilizadas en el cultivo de la vid y en el proceso de elaboración del vino persiguen esa misma finalidad: Una técnica que se difunde rápidamente es el cultivo de la vid en espaldera, que va sustituyendo al tradicional cultivo en vaso: en el año 2009 las plantaciones en espaldera representaban el 15 por ciento de la superficie del viñedo de la DOP Calatayud, el 41 por ciento de la de Cariñena y el 50 de la del Campo de Borja (Gobierno de Aragón); un buen indicador de la rapidez con que se extiende este sistema es que dos años antes en la DOP Cariñena ocupaba sólo el 30 por ciento del viñedo (Loscertales, 2009, p. 124). El nuevo sistema aumenta el rendimiento de las cepas, pues favorece una mayor insolación de toda la planta, facilita la mecanización de las tareas de cultivo y permite una vendimia más rápida.

La vid tradicionalmente se cultiva en secano, pero en los últimos años se está extendiendo el riego controlado por goteo, para asegurar a las plantas una aportación adicional de agua en el caso de que las lluvias sean muy escasas; con ello se pretende garantizar un rendimiento mínimo de las cepas. Actualmente existen instalaciones para esta práctica en el 8 por ciento del viñedo de Calatayud, el 24 por ciento del de Cariñena y el 30 por ciento del de Campo de Borja (Gobierno de Aragón). 
A todo lo expuesto cabe añadir que en las tres DOP se está generalizando el control científico de la vendimia, la cual se lleva a cabo en el momento óptimo de maduración de la uva; los vinos tradicionales de la zona se caracterizaban por una fuerte graduación alcohólica, adecuada cuando se buscaba en el vino energía para la realización de trabajos físicos duros; ahora normalmente la vendimia se hace en fechas más tempranas que antes, para obtener una menor concentración de azúcares en la uva y, con ello, una menor graduación alcohólica del vino. También están completamente generalizadas las técnicas de control de la fermentación y de las restantes fases del tratamiento del vino, con las que se garantiza cada vez mejor su calidad.

Atendiendo a los datos utilizados, se aprecia que las DOP de Cariñena y Campo de Borja han incorporado de manera importante las nuevas técnicas de cultivo, tanto la espaldera como el riego controlado, mientras que en la de Calatayud estas prácticas son todavía claramente minoritarias. Teniendo en cuenta esta situación contrastada entre las tres DOP zaragozanas en cuanto a técnicas de cultivo y la antes expuesta a partir de las variedades de uva, podemos afirmar la existencia de un modelo diferente para cada DOP: la de Calatayud es la más apegada a la tradición, tanto por el peso de la variedad de uva tradicional como por la escasa difusión de los nuevos métodos de cultivo. La de Cariñena es la menos vinculada a la tradición, como se deduce de la extensión que alcanzan las nuevas variedades y los nuevos métodos de cultivo, si bien no renuncia a la misma. En la de Campo de Borja es donde los nuevos métodos han alcanzado mayor difusión, pero la variedad tradicional de uva es la dominante, clara e intencionadamente.

\section{La conquista de nuevos mercados: la apuesta por la exportación}

La evolución del mercado nacional, con descenso del consumo y aumento de la competencia por la presencia de vinos foráneos, empuja a los diferentes actores económicos de este sector productivo a los mercados internacionales, a veces muy alejados geográficamente. Desde el inicio del presente siglo, contando con las oscilaciones normales de las cosechas y de las coyunturas comerciales, las exportaciones han seguido una tendencia creciente en Cariñena y Campo de Borja (figura 2). Cariñena es la que presenta los volúmenes más elevados, habiendo superado los 200 mil hectolitros en el año 2009; entre la campaña inicial y final de la serie el crecimiento ha sido del 111 por ciento. Campo de Borja, con unos volúmenes más reducidos, es, sin embargo, la que ha registrado una tasa de crecimiento más elevada, del 254 por ciento. Calatayud ha seguido una trayectoria creciente hasta el 2005, pero decreciente desde ese año, dando como balance final una reducción del 20 por ciento. 


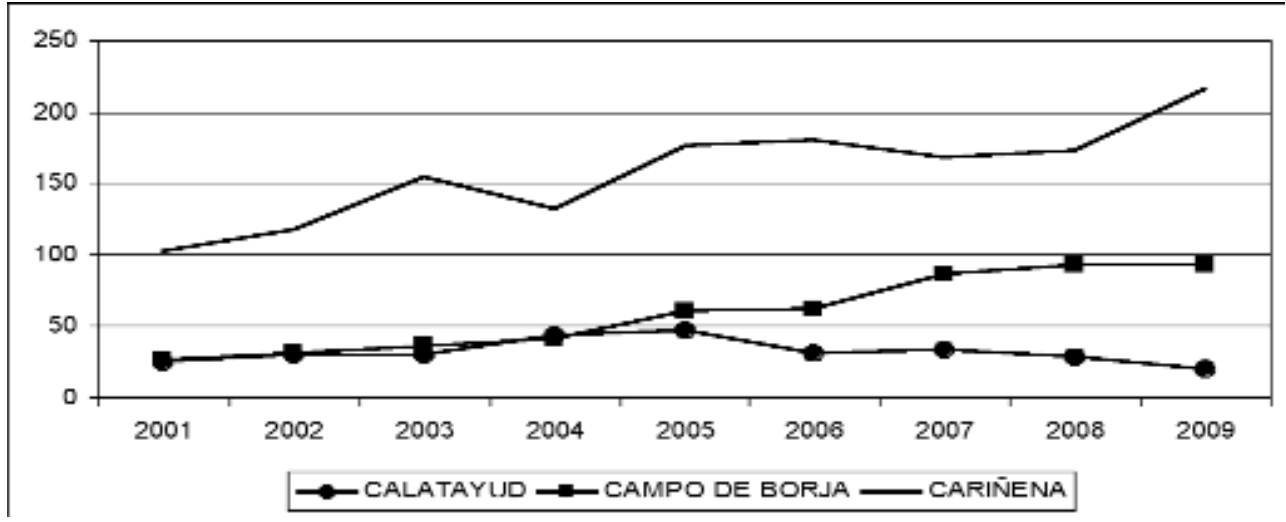

Fuente: Ministerio de Medio Ambiente y Medio Rural y Marino: Datos de los vinos de calidad producidos en regiones determinadas. Campaña 2008/2009 (y anteriores desde 2000-2001). http://www.mapa.es/alimentacion/pags/denominacion/documentos/INFORME\%202008-2009\%20def.pdf

Figura 2. Exportación de vino de las Denominaciones de Origen Protegidas de la provincia de Zaragoza (miles de hectolitros).

La proporción de vino exportado respecto al total de vino comercializado muestra también una tendencia creciente a lo largo del periodo: por DOP, en Cariñena ha pasado del 51 al 57 por ciento del total, en Campo de Borja del 45 al 68 por ciento y en Calatayud del 29 al 48. Las cifras indican que las dos primeras trabajan ya más para el mercado internacional que para el nacional, mientras que la de Calatayud está ya muy cerca de exportar la mitad de su producción. Conviene añadir que la totalidad del vino exportado desde las tres DOP es vino embotellado, habiendo desaparecido la exportación a granel, que en otro tiempo tuvo cierta importancia. El vino en botella, con su etiqueta correspondiente, que hace referencia a cada una de las DOP objeto de estudio, es el único que puede llegar a los consumidores de diferentes lugares del mundo. La participación en ferias, las misiones comerciales, el haber conseguido premios importantes o figurar en publicaciones de prestigio, favorecen la penetración en el mercado internacional.

La exportación de vino, como cabía esperar, se dirige principalmente a los países de la Unión Europea (UE): en la campaña 2000-2001 tanto en la DOP Calatayud como en la de Cariñena este mercado representaba aproximadamente el 90 por ciento de sus exportaciones, mientra que en la DOP Campo de Borja el porcentaje sólo alcanzó un 70 por ciento (tabla 1). Pero a lo largo del siglo las tres DOP han diversificado notablemente sus mercados internacionales, de manera que dichos porcentajes se han 
reducido significativamente, sobre todo en Calatayud, donde sólo representa en la actualidad un tercio del total de las mismas; en las otras dos DOP, a pesar de la reducción, el mercado comunitario sigue absorbiendo la mayor parte de las ventas al exterior.

Tabla 1. Distribución de las exportaciones de vino de las Denominaciones de Origen Protegidas de la provincia de Zaragoza por grandes áreas geográficas.

\begin{tabular}{|l|c|c|c|c|c|c|}
\hline \multirow{2}{*}{$\begin{array}{l}\text { Denominación de } \\
\text { Origen Protegida }\end{array}$} & \multicolumn{3}{|c|}{ 2000-2001 } & \multicolumn{3}{c|}{ 2008-2009 } \\
\cline { 2 - 7 } & $\begin{array}{c}\text { Total } \\
\text { (Hl.) }\end{array}$ & $\begin{array}{c}\text { \% Unión } \\
\text { Europea }\end{array}$ & $\begin{array}{c}\text { \% Resto } \\
\text { del mundo }\end{array}$ & $\begin{array}{c}\text { Total } \\
\text { (Hl. })\end{array}$ & $\begin{array}{c}\text { \% Unión } \\
\text { Europea }\end{array}$ & $\begin{array}{c}\text { \% Resto } \\
\text { del mundo }\end{array}$ \\
\hline Calatayud & 25.225 & 92,22 & 7,78 & 20.314 & 32,72 & 67,28 \\
Campo de Borja & 26.235 & 68,76 & 31,24 & 92.822 & 58,55 & 41,45 \\
Cariñena & 102.158 & 89,50 & 10,50 & 215.723 & 77,32 & 22,68 \\
\hline
\end{tabular}

Fuente: Ministerio de Medio Ambiente y Medio Rural y Marino: Datos de los vinos de calidad producidos en regiones determinadas. Campaña 2008/2009 (y 2000-2001).

http://www.mapa.es/alimentacion/pags/denominacion/documentos/INFORME\%202008-2009\%20def.pdf

Dentro de la UE los principales clientes a lo largo del siglo han sido Alemania y Reino Unido, ya que en ellos la producción de vino es muy baja o inexistente: las DOP Cariñena y Calatayud se han decantado claramente hacia el mercado alemán, que absorbió el 46 por ciento de las ventas comunitarias de ambas en la campaña 2008-2009; Campo de Borja, sin embargo, se ha volcado sobre todo en el mercado británico ( 55 por ciento en dicha campaña). Cabe resaltar, por lo difícil que es penetrar en los países tradicionalmente productores, la significativa presencia de los vinos de Cariñena en Francia desde 2005 (en la campaña 2008-2009 éste fue el cuarto país cliente de la UE).

En el mercado no comunitario destaca claramente Norteamérica: los Estados Unidos absorben el 84 por ciento de las ventas extracomunitarias de Calatayud y el 48 por ciento de las de Campo de Borja, que vende a Canadá otro 27 por ciento. La DOP Cariñena tiene un mercado más diversificado, pues las ventas destinadas a Norteamérica apenas superan la mitad del total de las extracomunitarias (28 por ciento a Estados Unidos, 23 por ciento a Canadá); en esta DOP cobran importancia algunos países europeos no comunitarios, como Suiza (tercer cliente, con el 19 por ciento), así como varios países del continente asiático, al que se destina el 13 por ciento del total de las exportaciones.

A modo de síntesis cabe afirmar que las tres DOP de la provincia de Zaragoza han asumido plenamente el reto de la internacionalización y de llevar sus vinos a los nue- 
vos consumidores, allá donde se encuentren. Para ello, a lo largo del siglo actual han desarrollado un esfuerzo creciente de exportación, dando respuesta a los retos del mercado exterior y a las dificultades crecientes experimentadas en el mercado interior (Escalona, Loscertales, Climent, 2009; Loscertales 2009). Actualmente el esfuerzo ha dado su fruto; las DOP de Cariñena y Campo de Borja destinan ya más de la mitad de su producción a los mercados internacionales; dentro de éstos destacan los de la UE, sobre todo el alemán y el británico, pero la búsqueda de nuevos clientes ha sido constante y se ha incrementado la proporción de vino exportado a mercados no comunitarios, destacando Norteamérica. Conviene señalar en este sentido que la DOP Cariñena se ha introducido con éxito, como ya se ha expuesto, en el mercado de uno de los grandes países productores europeos, Francia, así como en los mercados asiáticos, donde el vino aún puede considerarse como un producto exótico, pero cuyo consumo crece y se considera actualmente como un signo de prestigio social.

\section{La organización empresarial}

El tejido productivo de las DOP zaragozanas ha experimentado algunos reajustes a lo largo del presente siglo. Empezando por la fase agrícola, la entrada en vigor de la nueva Organización Común del Mercado está incentivando localmente los arranques de viñas; el envejecimiento de la población influye también negativamente en la evolución de la superficie de viñedo. Así ocurre en las DOP zaragozanas, aunque se observa que la superficie de viñedo inscrita ha evolucionado de manera diferente en cada una de ellas (figura 3): el viñedo de Cariñena se ha mantenido estable en torno a las 16.000 hectáreas, excepto en los años inicial y final de la serie, en los que se situó en torno a las 15.000; en el futuro próximo se verá si el descenso de la última campaña es un reajuste circunstancial o el inicio de una tendencia decreciente. Campo de Borja se ha mantenido estable, por encima de las 7.000 hectáreas, mientras que Calatayud ha experimentado un retroceso equivalente al 45 por ciento de la extensión de principios de siglo, fecha en la que superaba ligeramente a Campo de Borja, mientras que ahora queda claramente por debajo.

En cuanto al número de viticultores, se aprecia en las tres DOP un periodo inicial de estabilidad, seguido en los últimos años de una brusca inflexión decreciente (figura 3): en Campo de Borja y Cariñena ésta se produce en la campaña 2006-2007, mientras que en Calatayud se retrasa hasta la de 2008-2009; el descenso ha sido del 36,46 y 62 por ciento, respectivamente, lo cual pone de manifiesto que el reajuste en el aparato productivo vitícola ha sido mucho más acusado en Calatayud que en las restantes DOP. La superficie media de las explotaciones se ha incrementado sensiblemente después del fuerte descenso del número de agricultores: de 2,5 a 3,6 hectáreas en Calatayud, de 3,6 a 5,7 en Campo de Borja y de 4,1 a 7,6 en Cariñena. 
Por lo que respecta a la producción vinícola, el número de bodegas inscritas en las DOP ha experimentado un crecimiento muy ligero entre 2001 y 2009: en Cariñena ha pasado de 50 a 55, en Campo de Borja de 15 a 17 y en Calatayud de 14 a $16^{3}$. En Campo de Borja y Calatayud las tres cuartas partes de las bodegas son embotelladoras, mientras que en Cariñena lo es el 56 por ciento. La gran mayoría de las bodegas son empresas de pequeño tamaño: sólo un número reducido de las mismas tiene un volumen de negocio superior a 6 millones de euros (figura 4). La DOP Campo de Borja dispone de un grupo significativo de empresas de tamaño medio. En la de Calatayud la forma jurídica dominante es la persona física, es decir, el pequeño empresario individual. Las cooperativas juegan un papel importante, sobre todo en la DOP de Cariñena, donde son empresas muy fuertes y controlan una parte muy significativa de la producción. ${ }^{4}$

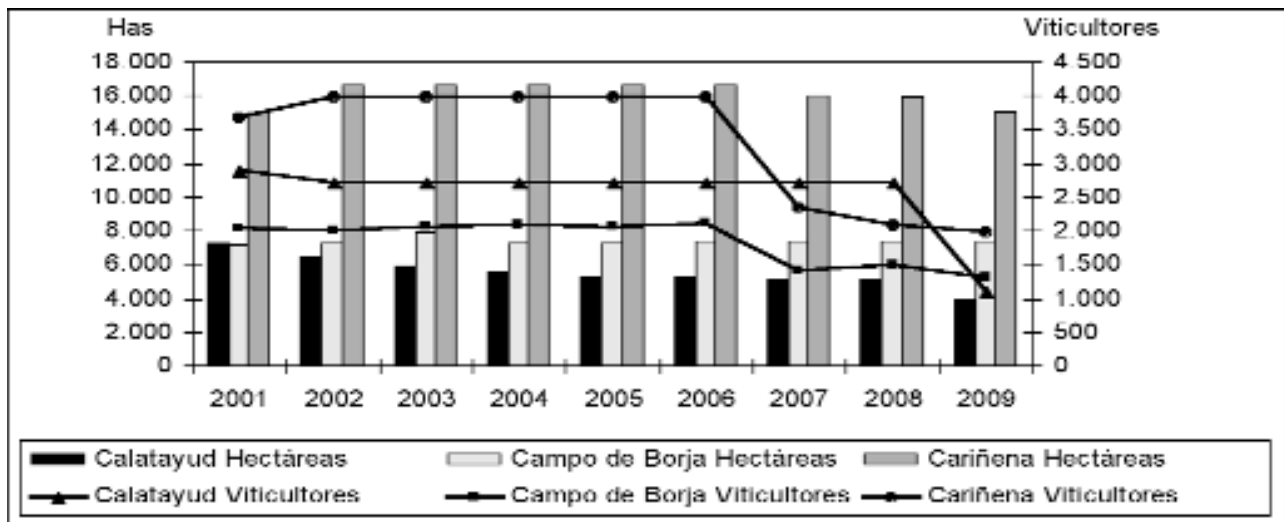

Fuente: Ministerio de Medio Ambiente y Medio Rural y Marino: Datos de los vinos de calidad producidos en regiones determinadas. Campaña 2008/2009 (y anteriores desde 2000-2001). http://www.mapa.es/alimentacion/pags/denominacion/documentos/INFORME\%202008-2009\%20def.pdf

Figura 3. Superficie de viñedo y número de viticultores de las Denominaciones de Origen Protegidas de la provincia de Zaragoza.

\footnotetext{
${ }^{3}$ Fuente: Ministerio de Medio Ambiente y Medio Rural y Marino: Datos de los vinos de calidad producidos en regiones determinadas. Campaña 2007/2008 y anteriores desde 2000-2001.

${ }^{4}$ Una sola de las cooperativas de Cariñena (Bodegas San Valero) controla a través de sus socios 3.500 hectáreas, es decir, el 22 por ciento de la superficie de viñedo de la DOP.
} 


\section{Conclusiones}

El artículo detalla en sus diferentes apartados los retos que tiene planteados el sector vinícola de las tres DOP zaragozanas, a los que los diferentes actores han debido dar respuesta adecuada mediante la adopción de diversos cambios: en los productos, para adaptarse a unos consumidores cada vez más exigentes y diversos; en los métodos de producción, tanto en el proceso vitícola como vinícola; en el mercado, cada vez más globalizado y en el que cabe destacar la aparición de nuevos países productores, lo que representa la llegada a la escena mundial de una nueva fuente de suministro; y, por último, en la organización industrial. Como se ha expuesto, las respuestas de las tres DOP zaragozanas han sido diferentes y ello explica su evolución y los contrastes existentes entre ellas.

El balance del conjunto del estudio realizado en las tres DOP zaragozanas permite afirmar la diferente evolución de la DOP Calatayud con respecto a las otras dos. Las exportaciones, aunque casi alcanzan en la actualidad la mitad del volumen de vino comercializado, han ido cayendo en los últimos años, sin que haya podido consolidarse la tendencia creciente de las primeras cinco campañas del siglo. Paralelamente ha experimentado una fuerte reducción de la superficie cultivada y del número de viticultores, en lo que parece un duro proceso de ajuste. La marcada atomización del tejido productivo (explotaciones vitícolas de pequeño tamaño y el predominio de entidades empresariales propiedad de personas físicas, pequeños bodegueros individuales y no sociedades), podría explicar esta situación y ser uno de los factores condicionantes del fuerte apego a la tradición, tanto en las variedades de uva como en los métodos de cultivo.

En la DOP Campo de Borja, la superficie cultivada se ha mantenido estable, a diferencia de la anteriormente citada, aunque también en ella se ha reducido el número de viticultores, si bien en menor proporción que en las restantes. Por otra parte, ha experimentado un fuerte incremento de las exportaciones, respondiendo al reto de la conquista de nuevos mercados. Presenta un tejido productivo menos atomizado, con explotaciones agrarias más grandes y, sobre todo, con una proporción relativamente alta de bodegas de tamaño medio. La estrategia productiva que han seguido viticultores y bodegas ha consistido en potenciar la variedad de uva autóctona (sin renunciar por completo a las foráneas) e introducir nuevas técnicas de cultivo, estrategia que ha dado los buenos resultados señalados.

La DOP Cariñena ha evolucionado también favorablemente en cuanto al crecimiento de las exportaciones y la conquista progresiva de nuevos mercados. La superficie vitícola cultivada presenta valores estables, mientras que el número de viticultores se ha reducido. Su tejido productivo se caracteriza por la existencia de explotaciones vitícolas de tamaño medio y por grandes bodegas cooperativas, que 


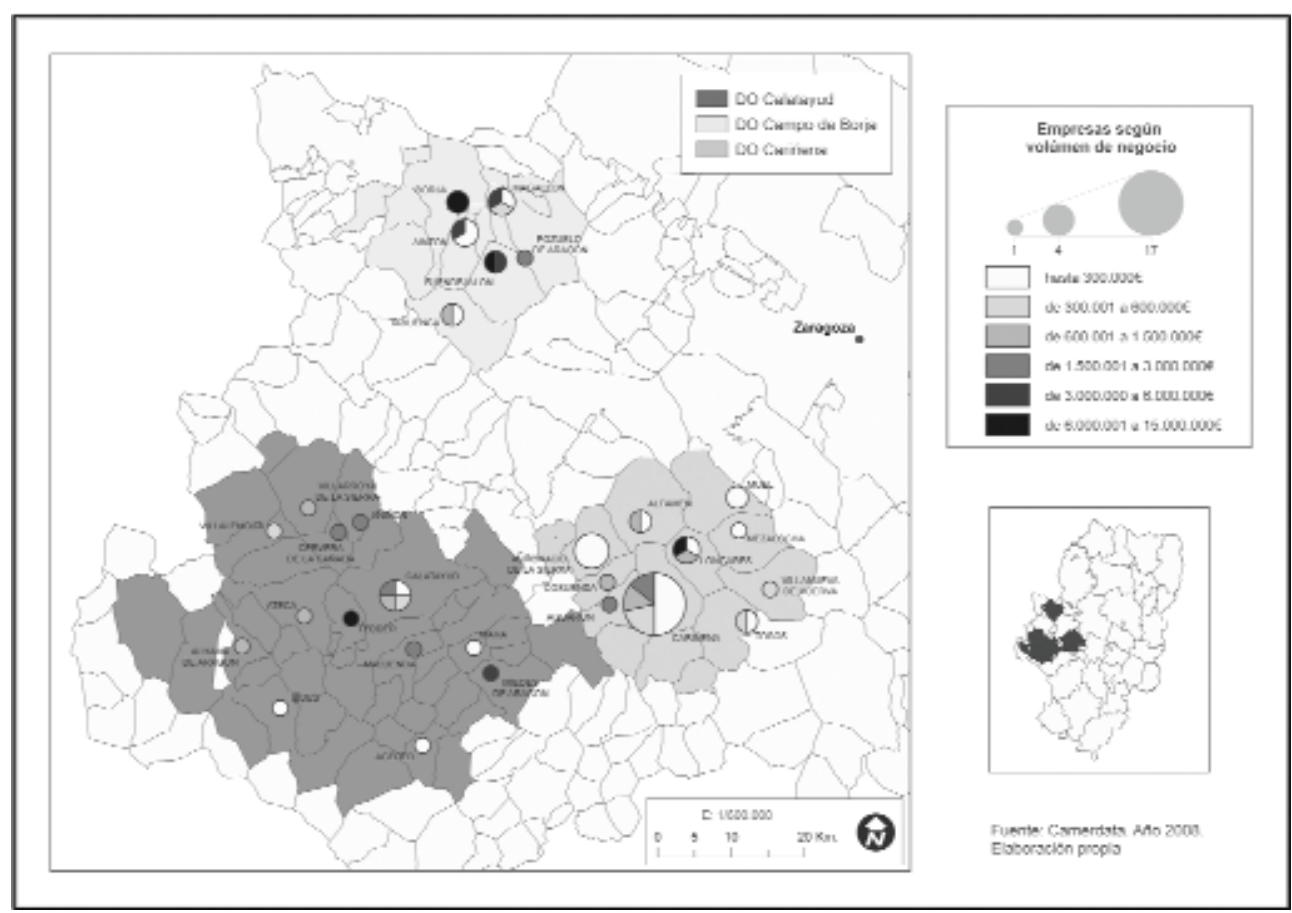

Fuente: CAMERDATA y Escalona, Loscertales y Climent (2009).

Figura 4. Localización y distribución por tamaño de las bodegas de las Denominaciones de Origen Protegidas de la provincia de Zaragoza (2008).

contrastan con las nuevas bodegas de dimensiones más reducidas, generalmente sociedades limitadas, que han venido apareciendo en época reciente (Loscertales, 2009). La estrategia aplicada ha consistido en diversificar el viñedo con nuevas especies y paralelamente los vinos, con los caldos obtenidos en su totalidad o en parte de las nuevas variedades, sin que ello suponga renunciar a las tradicionales; paralelamente se han incorporado de forma masiva las nuevas técnicas de cultivo. Ello permite decir que ha sabido conjugar la tradición con la innovación y ésa es la base de su evolución reciente.

Potenciar la tradición, como se hace en las DOP de la provincia de Zaragoza, es considerada una estrategia ganadora: "a menudo se piensa que los alimentos de un origen local claro son de mayor calidad que los alimentos globales" (Murdoch y Miele, 1999, p. 469); en el caso del vino, los consumidores otorgan su confianza al origen local y a los modos tradicionales de producción (Lindkvist y Sánchez, 2008), 
lo cual además viene avalado por la existencia de una DOP. Las DOP Cariñena y Campo de Borja, aun siguiendo estrategias productivas diferentes, coinciden en sus buenos resultados, como indican el incremento de las exportaciones y el mantenimiento de la superficie vitícola; la clave de su trayectoria posiblemente esté en la solidez de sus tejidos productivos. La DOP Calatayud, fuertemente orientada a la exportación y con la mayor presencia relativa en mercados no europeos, afronta un ajuste encaminado a reforzar su potencial productivo. Por todo ello, cabe afirmar que el sector vitivinícola tiene un futuro prometedor $y$, en consecuencia, va a seguir desempeñando un importante papel en el desarrollo local de los territorios de las tres DOP estudiadas.

\section{Bibliografía}

Albisu Aguado, L.M. (2005). La industria agroalimentaria y su necesidad de profesionales especializados. Agricultura. Revista agropecuaria, 873, 288-290.

Barco Royo, E. (2007). Denominaciones de origen. Distribución y consumo, 96, 27-41.

Barco Royo, E., Pinillos García, M.O. y Navarro Pérez, M.C. (2006). Estrategias de diversificación versus estrategias de tamaño. Cuaderno de Campo, 34, 12-16.

Escalona Orcao, A.I., Loscertales Palomar, B. y Climent López, E. (2009). Nuevos retos para el desarrollo territorial: los espacios del vino y del calzado en la provincia de Zaragoza (en línea). Zaragoza, Diputación Provincial de Zaragoza. <http://www.dpz. es/diputacion/areas/presidencia/4espacio/documentos/premios/nuevos_retos_te rritorial09.pdf> (Consulta: 06.08.2010)

Escalona Orcao, A.I., Loscertales Palomar, B. y Climent López, E. (2010). Nuevos retos empresariales en las áreas rurales. Convenciones y estrategias de las industrias del vino y del calzado en la provincia de Zaragoza. Comunicación presentada al
XII Congreso Ibérico de Geografía, Oporto, octubre de 2010 (en prensa).

Hall, P. y Preston, P. (1990). La ola portadora. Nuevas tecnologías de la información y geografia de las innovaciones 1846-2003. Madrid, FUNDESCO.

Lindkvist, K.B. y Sánchez, J.L. (2008). Conventions and innovation: A comparison of two localised natural resourcebased industries. Regional Studies, 42 (3), 343-354.

Loscertales Palomar, B. (2009). La Denominación de Origen Cariñena: una apuesta por la singularidad y la calidad en un mundo globalizado. Zaragoza, Ediciones 94

Murdoch, J. y Miele, M. (1999). 'Back to Nature': changing 'worlds of production' in the food sector. Sociologia Ruralis 39, pp. $465-483$.

Ponte, S. (2009). Governing trough quality: conventions and supply relations in the value chain for South African wine. Sociologia Ruralis, 49 (3), pp. 236-257. 